\title{
PERFORACIONES ESCALARIFORMES EN VASOS DE ÁRBOLES NATIVOS DE CHILE
}

\author{
SCALARIFORM PERFORATION PLATES IN THE VESSELS \\ OF CHILEAN WOODS
}

\author{
Mónica RALLO $^{1}$; Dayana MONTECINOS ${ }^{1}$; Tania MUNDACA ${ }^{1}$ \\ ${ }^{1}$ Departamento de Ingeniería de la Madera, Facultad de Ciencias Forestales, Universidad de \\ Chile. Santiago-Chile. \\ Autor para correspondencia: mrallo@uchile.cl
}

Recibido: 25.06.2007. Aceptado: 12.07.2008

\section{RESUMEN}

Las placas de perforación son aberturas que se encuentran ubicadas en los extremos de los elementos vasos, permitiendo el paso de fluidos de un vaso a otro en dirección longitudinal. Existen varios tipos de placas de perforación, simples, escalariformes, reticuladas, cribosas y combinaciones de placas.

En este trabajo se realizó una descripción y clasificación de las perforaciones escalariformes, como un aporte al conocimiento de la estructura anatómica de las maderas nativas. Determinándose el número de barras en éstas y el espaciamiento entre ellas, para esto se seleccionaron 10 especies de Angiospermas nativas de Chile que presentaban este tipo de perforación en sus vasos.

Las especies analizadas fueron Aextoxicon punctatum Ruiz et Pavón, Laureliopsis philippiana (Looser) Schodde, Laurelia sempervirens (Ruiz et Pavón) Tul., Weinmannia trichosperma Cav., Caldcluvia paniculata (Cav.) D. Don., Eucryphia cordifolia Cav., Citronella mucronata (Ruiz et Pavón) D. Don, Luma apiculata (DC.) Burret, Escallonia revoluta (Ruiz et Pavón) Pers., Azara serrata Ruiz et Pavón.

Palabras claves: Placas de perforación, escalariformes, anatomía de la madera, clasificación

\begin{abstract}
Perforation plates are openings in the end walls of vessel elements, allowing water flow between neighboring vessel elements in longitudinal direction. There are many types of perforation plates, simple, scalariform, reticulate and foraminate.

In this study a classification and description of scalariform plates was made, as a contribution to the knowledge of anatomic wood structure of native species. To determine the number of bars and spaces between bars per scalariform perforation plate, were selected 10 species of natives Angiosperm of Chile having this type perforation.

Species were Aextoxicon punctatum Ruiz et Pavón, Laureliopsis philippiana (Looser) Schodde, Laurelia sempervirens (Ruiz et Pavón) Tul., Weinmannia trichosperma Cav., Caldcluvia paniculata (Cav.) D.Don., Eucryphia cordifolia Cav., Citronella mucronata (Ruiz et Pavón) D. Don, Luma apiculata (DC.) Burret, Escallonia revoluta (Ruiz et Pavón) Pers., Azara serrata Ruiz et Pavón.
\end{abstract}

Keywords: Perforation plates, scalariform, wood anatomy, classification 


\section{INTRODUCCIÓN}

Las placas de perforación son aberturas que se encuentran ubicadas generalmente en el extremo de los elementos vasos de las Angiospermas, permitiendo el paso de fluidos, en dirección longitudinal de un segmento de vaso a otro. Estas perforaciones envuelven a todas las capas de la pared celular, lo que las diferencia de las punteaduras que tienen una estructura más compleja. En la diferenciación celular, mediante procesos enzimáticos las paredes terminales entre los vasos pueden desaparecer por completo o quedar restos de estas paredes dando origen a diversos tipos de perforaciones. También procesos mecánicos, que ocurren durante este periodo, pudieran estar involucrados en la formación de las perforaciones (Meylan y Butterfield 1975; Panshin y Zeeuw 1980; Díaz-vaz 2003).

Entre los diferentes tipos de perforaciones las más comunes son las simples, que están presentes en el $80 \%$ de las maderas del mundo, éstas consisten de una sola gran abertura redondeada u ovalada; las perforaciones escalariformes consisten de aberturas alargadas y paralelas separadas por una o varias barras no ramificadas; las reticuladas formadas por aberturas separadas por restos de paredes que presentan la apariencia de una red y las perforaciones foraminadas o cribosas con múltiples aberturas circulares o elípticas. Además algunas especies pueden presentar más de un tipo de perforación en sus vasos y combinaciones de placas. (Meylan y Butterfield 1975; IAWA Committee 1989)

Las perforaciones escalariformes han sido motivo de numerosos estudios relacionados con la evolución de las especies; varios autores como Boureau (1957), Carlquist (1961,1988), Ellerby y Ennos (1998) y Jansen et al. (2004) mencionan que estas perforaciones son primitivas y las perforaciones simples más evolucionadas. Esto se relaciona con la longitud y diámetro de los elementos vasos que presentan este tipo de perforaciones, los de gran longitud y diámetros pequeños generalmente presentan perforaciones escalariformes con numerosas barras; los cambios que ocurren en los vasos y sus perforaciones, pasan por estados intermedios como la disminución de su longitud y diámetro, reducción del número de barras y un mayor distanciamiento entre las aberturas de las perforaciones, hasta transformarse en vasos cortos y de gran diámetro con perforaciones simples.

En términos ecológicos se ha observado que las especies localizadas en zonas de climas fríos, presentan una alta incidencia de perforaciones escalariformes, mientras que las especies localizadas en zonas con climas secos y altas temperaturas, se caracterizan por la presencia casi exclusiva de perforaciones simples. Esto estaría relacionado con funciones de adaptación de las especies (Carlquist 1988; Baas y Wheeler 1996; Segala y Angyalossy-Alfonso 2000; Hacke y Sperry 2001; Jansen et al. 2004).

Estudios sobre la funcionalidad de las perforaciones escalariformes con respecto al movimiento de agua, han demostrado que estas oponen mayor resistencia al paso de éste y tienen una significativa tarea atrapando las burbujas de aire que se desarrollan en los vasos, evitando de esta forma problemas de embolismo (Carlquist 1988; Ellerby y Ennos 1998; Schulte 1999).

Las perforaciones escalariformes presentan diferencias en sus formas, número de barras y espaciamiento entre éstas, lo que es de mucha utilidad en la identificación de maderas. Las familias correspondientes a las especies de este estudio son mencionadas en los trabajos de Boureau (1957), Meylan y Butterfield (1975), Metcalfe y Chalk (1983) y Carlquist (1988), por la presencia de este tipo de perforaciones.

El presente trabajo tiene como objetivo realizar una descripción y clasificación de las perforaciones escalariformes, como un aporte al conocimiento de la estructura anatómica de las maderas nativas. 


\section{MATERIALES Y MÉTODOS}

El material de estudio corresponde a muestras de maderas pertenecientes a la Xiloteca del Laboratorio de Anatomía de la Madera de la Facultad de Ciencias Forestales, Universidad de Chile. Se seleccionaron 10 especies nativas que presentaban placas de perforación escalariformes. (Tabla 1)

Las maderas se obtuvieron de árboles adultos y troncos principales, los cortes microscópicos y maceraciones se realizaron según métodos estándar. (Normand 1972).

Tabla 1: Lista de las especies estudiadas y número de muestra en Xiloteca

\begin{tabular}{|c|c|c|c|}
\hline FAMILIA & ESPECIE & $\begin{array}{l}\text { NOMBRE } \\
\text { COMÚN }\end{array}$ & $\begin{array}{l}\text { MUESTRA DE } \\
\text { XILOTECA }\end{array}$ \\
\hline Aextoxicaceae & Aextoxicon punctatum Ruiz et Pavón & Olivillo & X003 \\
\hline Cunoniaceae & $\begin{array}{l}\text { Weinmannia trichosperma Cav. } \\
\text { Caldcluvia paniculata (Cav.) D. Don. }\end{array}$ & $\begin{array}{l}\text { Tineo } \\
\text { Tiaca }\end{array}$ & $\begin{array}{r}\mathrm{X} 038 \\
\mathrm{X} 008\end{array}$ \\
\hline Escalloniaceae & Escallonia revoluta (Ruiz et Pavón) Pers. & Lun & $\mathrm{X} 042$ \\
\hline Eucryphiaceae & Eucryphia cordifolia Cav. & Ulmo & $\mathrm{X} 016$ \\
\hline Flacourtiaceae & Azara serrata Ruiz et Pavón & Corcolén & X044 \\
\hline Icacinaceae & Citronella mucronata (Ruiz et Pavón) D. Don. & Hullipatagua & X043 \\
\hline Monimiaceas & $\begin{array}{l}\text { Laureliopsis philippiana (Looser) Schodde } \\
\text { Laurelia sempervirens (Ruiz et Pavón) Tul. }\end{array}$ & $\begin{array}{l}\text { Tepa } \\
\text { Laurel }\end{array}$ & $\begin{array}{r}\text { X019 } \\
\text { X039 }\end{array}$ \\
\hline Myrtaceae & Luma apiculata (DC.) Burret & Arrayán & X021 \\
\hline
\end{tabular}

Se determinó el número de barras en las perforaciones escalariformes observadas y se clasificaron según la lista estándar IAWA (1989), de acuerdo a las siguientes categorías: $\leq 10$ barras, $10-20$ barras, 20 -40 barras y $\geq 40$ barras. El espaciamiento entre las barras se midió en $\mu \mathrm{m}$. Fueron examinados entre 20 a 25 elementos vasos por especie, en cortes longitudinales radiales y maceraciones; se obtuvieron imágenes digitales con un microscopio óptico con cámara de video incorporada, para mostrar las diferentes perforaciones escalariformes, presentes en las especies estudiadas y microfotografías en microscopio electrónico de barrido (SEM) de Aextoxicon punctatum. 


\section{RESULTADOS Y DISCUSIÓN}

\section{Número de barras en las perforaciones}

Aextoxicon punctatum y Laureliopsis philippiana presentaron las perforaciones escalariformes con el mayor número de barras, las que fluctuaron entre 20 y 70 (Fig.1); Rancusi et al. (1987) observó 38 a 100 barras en las perforaciones de Aextoxicon punctatum y 25 a 70 barras en Laureliopsis philippiana. En Weinmannia trichosperma, Caldcluvia paniculada, Eucryphia cordifolia y Luma apiculata las perforaciones mostraron entre 10 a 34 barras (Fig.1), mientras que Rancusi et al. (1987) observó 9 a 47 barras en las perforaciones de estas especies.
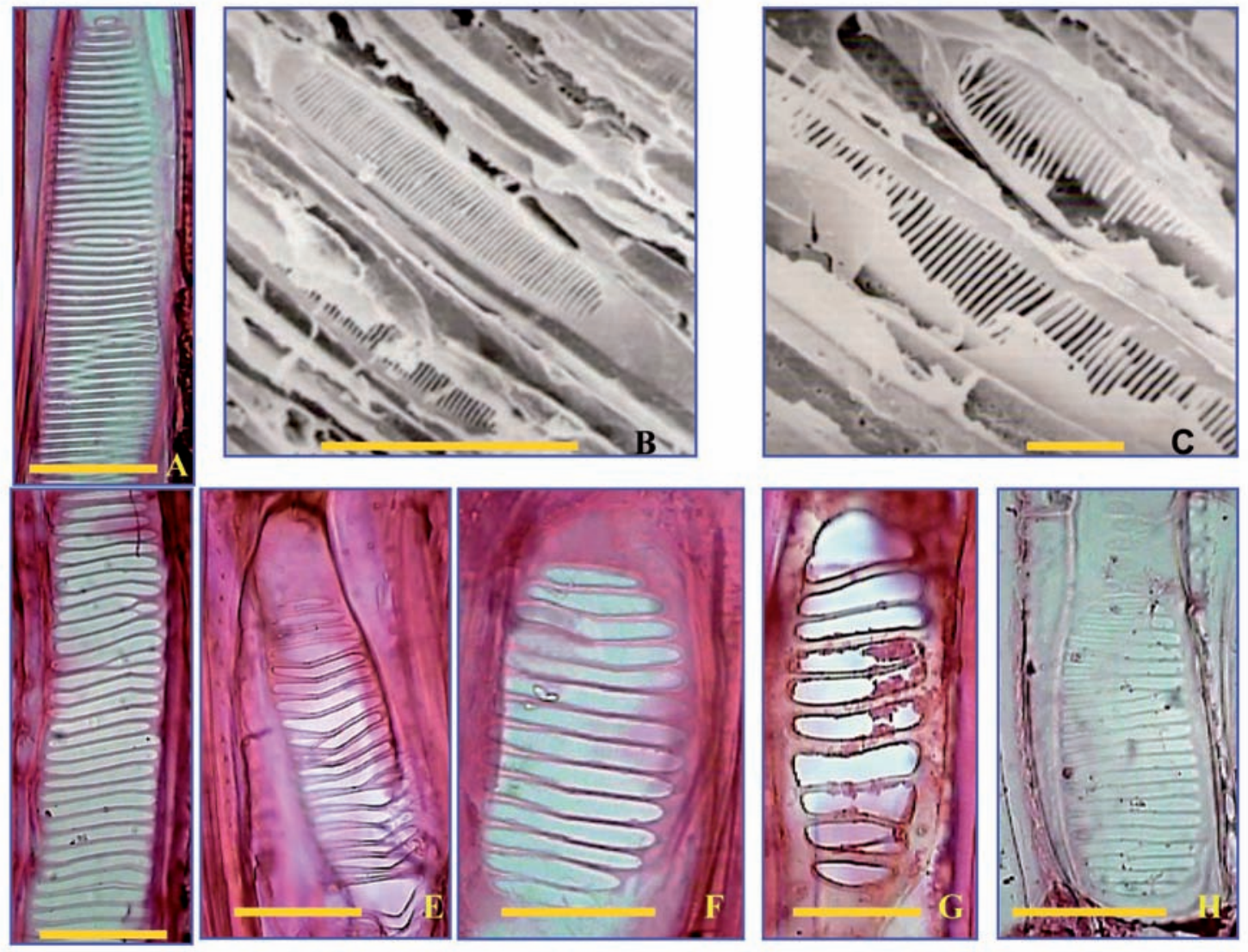

Figura 1: Perforaciones escalariformes en cortes radiales. A: Aextoxicon punctatum; B y C: Aextoxicon punctatum (SEM); D: Laureliopsis philippiana; E: Weinmannia trichosperma; F: Caldcluvia paniculata; G: Eucryphia cordifolia; H: Luma apiculata. Escalas: A, D, E, $\mathrm{G}=25 \mu \mathrm{m} ; \mathrm{B}=50 \mu \mathrm{m} ; \mathrm{C}=20 \mu \mathrm{m} ; \mathrm{F}$ y $\mathrm{H}=30 \mu \mathrm{m}$.

La especie Laurelia sempervirens (Fig.2) presentó perforaciones con 4 a 17 barras, mientras que Rancusi et al. (1987) observó 11 a 21 barras en esta especie. Citronella mucronata y Escallonia revoluta (Fig.2) presentaron perforaciones con 6 a 17 barras. En Azara serrata (Fig.2) se observaron las perforaciones con menor número de barras 3 a 8, mientras que Rancusi et al. (1987) encontró 1 a 3 barras en Azara lanceolata y A. integrifolia. 
Con respecto a las especies Escallonia revoluta y Azara serrata que presentaron las perforaciones escalariformes con el menor número de barras, éstas pertenecen a las familias Escalloniaceae y Flacourtiaceae respectivamente, mencionadas en Boureau (1957), Metcalfe y Chalk (1983) y Carlquist (1988) por la presencia de perforaciones escalariformes con un rango en número de barras de 1 a 20, independiente de su hábito de crecimiento arbóreo o arbustivo.
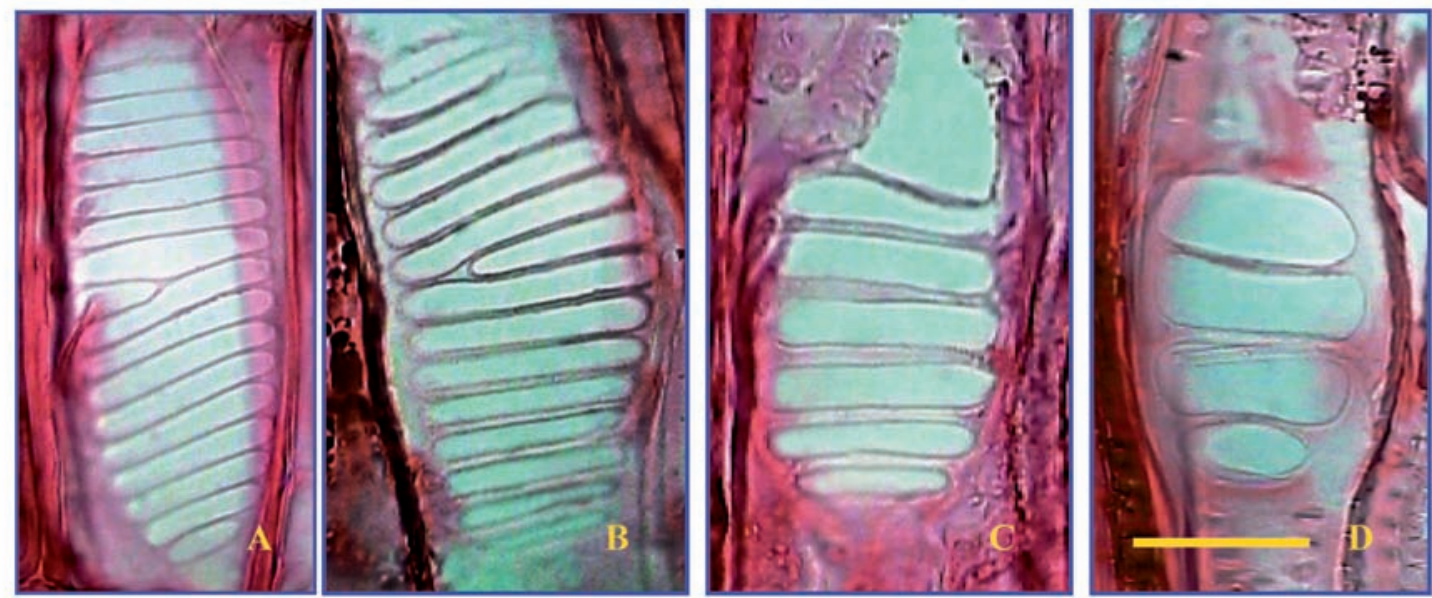

Figura 2: Perforaciones escalariformes en cortes radiales. A: Laurelia sempervirens; B: Citronella mucronata; C: Escallonia revoluta; D: Azara serrata. Escala $=30 \mu \mathrm{m}$

\section{Espaciamiento entre las barras}

La especie que presentó el espaciamiento más estrecho entre sus barras fue Aextoxicon punctatum (Fig. 1. A, B y C), con 1 a $2 \mu \mathrm{m}$; el resto de las especies analizadas tienen entre 2 a $6 \mu \mathrm{m}$ de espaciamiento en sus perforaciones. En Azara serrata (Fig. 2. D) se observaron los espaciamientos más anchos 6 a $11 \mu \mathrm{m}$ (Tabla 2). Al respecto Boureau (1957) señala que los espacios entre las barras menores o iguales a $6 \mu \mathrm{m}$ serían estrechos y los superiores anchos.

Las perforaciones escalariformes observadas con mayor número de barras, presentaron espaciamientos estrechos, mientras que las perforaciones escalariformes con menor número de barras presentaron espaciamientos más anchos entre sus barras, coincidiendo con estudios relacionados con evolución de las especies. (Boureau 1957; Carlquist 1961,1988; Ellerby y Ennos 1998; Jansen et al. 2004).

Otros autores como Wagemann (1949), Carlquist (1988), Diaz-vaz et al. (2002) y Diaz-vaz (2003) que describen la estructura anatómica de varias de las especies en estudio, mencionan que estas presentan perforaciones escalariformes con numerosas barras, pero no determinan número ni espaciamiento entre sus barras.

Los resultados obtenidos para las perforaciones escalariformes se muestran en la Tabla 2, especificando el número de barras y el espaciamiento entre éstas. 
Tabla 2: Resultados obtenidos

\begin{tabular}{|l|c|c|c|c|}
\hline \multicolumn{1}{|c|}{ Especie } & \multicolumn{2}{|c|}{ Número de barras } & Rango & Promedio \\
\cline { 2 - 5 } & Rango & Promedio & $1-2$ & 1,5 \\
\hline Aextoxicon punctatum & $36-70$ & 49 & $2-4$ & 3 \\
Laureliopsis philippiana & $20-46$ & 38 & $2-6$ & 3 \\
Eucryphia cordifolia & $14-28$ & 25 & $2-4$ & 3 \\
Luma apiculata & $15-24$ & 20 & & 4 \\
Weinmannia & $15-34$ & 18 & $3-5$ & 4,5 \\
trichosperma & $10-31$ & 18 & $4-5$ & 3,5 \\
Caldcluvia paniculata & $9-17$ & 13 & $3-4$ & 5 \\
Citronella mucronata & $4-17$ & 13 & $4-6$ & 4,5 \\
Laurelia sempervirens & $6-14$ & 9 & $4-5$ & 8,5 \\
\hline Escallonia revoluta & $3-8$ & 6 & $6-11$ & \\
Azara serrata & & & & \\
\hline
\end{tabular}

De acuerdo al número de barras, cada especie fue clasificada mediante la siguiente tabla basada en las categorías de IAWA (1989)

Tabla 3: Clasificación del número de barras obtenidas, según IAWA (1989)

\begin{tabular}{|l|c|c|}
\hline \multicolumn{1}{|c|}{ Especie } & Rangos de resultados & Categorías según IAWA (1989) \\
\hline Aextoxicon punctatum & $36-70$ & $\geq 40$ barras \\
Laureliopsis philippiana & $20-46$ & $20-40$ barras \\
\hline Eucryphia cordifolia & $14-28$ & $20-40$ barras \\
Luma apiculata & $15-24$ & $10-20$ barras \\
Weinmannia trichosperma & $15-34$ & $10-20$ barras \\
Caldcluvia paniculata & $10-31$ & $\leq 10$ barras \\
\hline Citronella mucronata & $9-17$ & $\leq 10$ barras \\
Laurelia sempervirens & $4-17$ & $6-14$ \\
\hline Escallonia revoluta & $3-8$ & \\
\hline Azara serrata & & \\
\hline
\end{tabular}




\section{Alteraciones en las perforaciones}

Algunas de las especies examinadas, presentaron modificaciones en la estructura de sus perforaciones escalariformes, como bifurcaciones o ramificaciones de sus barras, las que se observaron por ejemplo en Laureliopsis philippiana, Laurelia sempervirens y Caldcluvia paniculata (Fig.3.A-B-C). ); Carlquist (1988), menciona que en la mayoría de las especies con placas de perforación escalariformes ocurren pequeñas aberraciones en sus barras, con forma de tenedor. Estas fueron también observadas por Schneider y Carlquist (2001).

Aextoxicon punctatum y Weinmannia trichosperma presentaron en algunas perforaciones, restos de paredes entre las barras muy similares a los observados por Meylan y Butterfield (1975) y Carlquist (1988), que indican que estos podrían corresponder a paredes primarias, o restos de celulosa que no se disolvieron en el proceso de lisis de las paredes terminales de los vasos. (Fig.3. D-E)

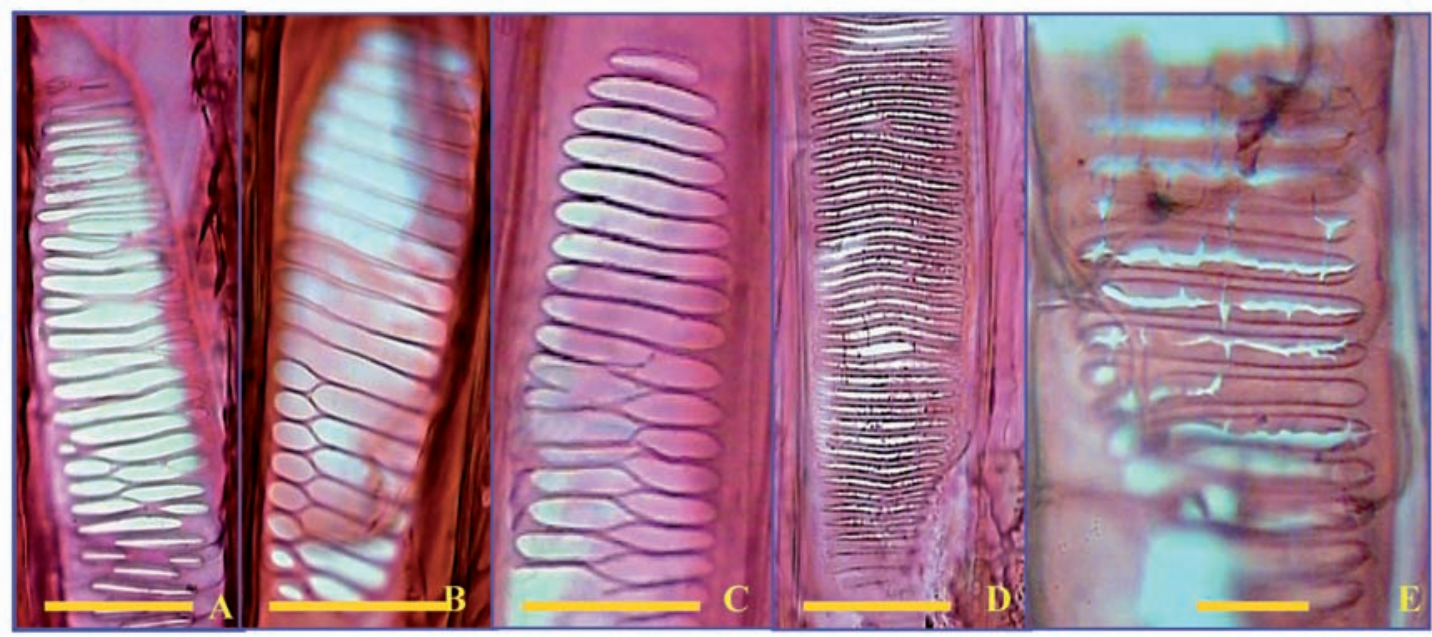

Figura 3: Perforaciones con modificaciones. A: Laureliopsis philippiana; B: Laurelia sempervirens; C: Caldcluvia paniculata. Restos de paredes en las perforaciones. D: Aextoxicon punctatum; E: Weinmannia trichosperma. Escalas: A y D $=25 \mu \mathrm{m}$; B y $\mathrm{C}=30 \mu \mathrm{m} ; \mathrm{E}=20 \mu \mathrm{m}$

\section{Presencia de otros tipos de perforaciones}

En Eucryphia cordifolia (Fig.4) se observaron placas escalariformes, simples y reticuladas en sus vasos. Wagemann (1949), Dickison (1978) y Rancusi (1987) encontraron sólo perforaciones simples y escalariformes en esta especie; mientras que Diaz-vaz (2003) menciona la presencia de los tres tipos de perforaciones en esta especie. 


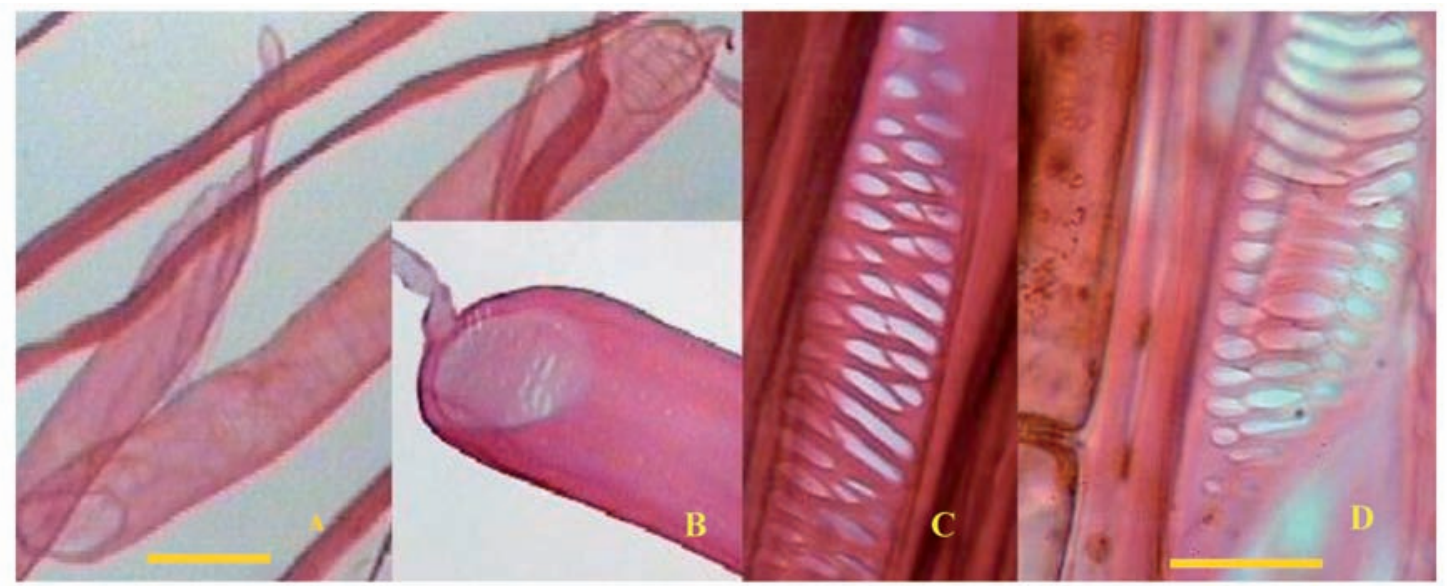

Figura 4: Perforaciones en Eucryphia cordifolia. A: Elemento vaso en maceración con perforación simple y escalariforme Escala $=150 \mu \mathrm{m}$; B: Perforación simple de un elemento vaso en una maceración; C - D: Perforaciones reticuladas en cortes radiales. Escala $=25 \mu \mathrm{m}$

Una combinación de placa de perforación fue observada en Laurelia sempervirens (Fig.5.A), que presentó una placa de perforación escalariforme en la pared terminal de un vaso y simple en el vaso adyacente, similar a las perforaciones encontradas por Meylan y Butterfield (1975) y Rudall (1982).

También se encontraron perforaciones con variadas formas en Laurelia sempervirens y Laureliopsis philippiana (Fig.5. B y C), este tipo de placas multiperforadas son mencionadas por Metcalfe y Chalk (1983), quien las describe como aberraciones y divergencias relacionadas con las puntuaciones, éstas no tendrían importancia taxonómica ya que se encuentran esporádicamente y están limitadas a muy pocas familias y géneros.
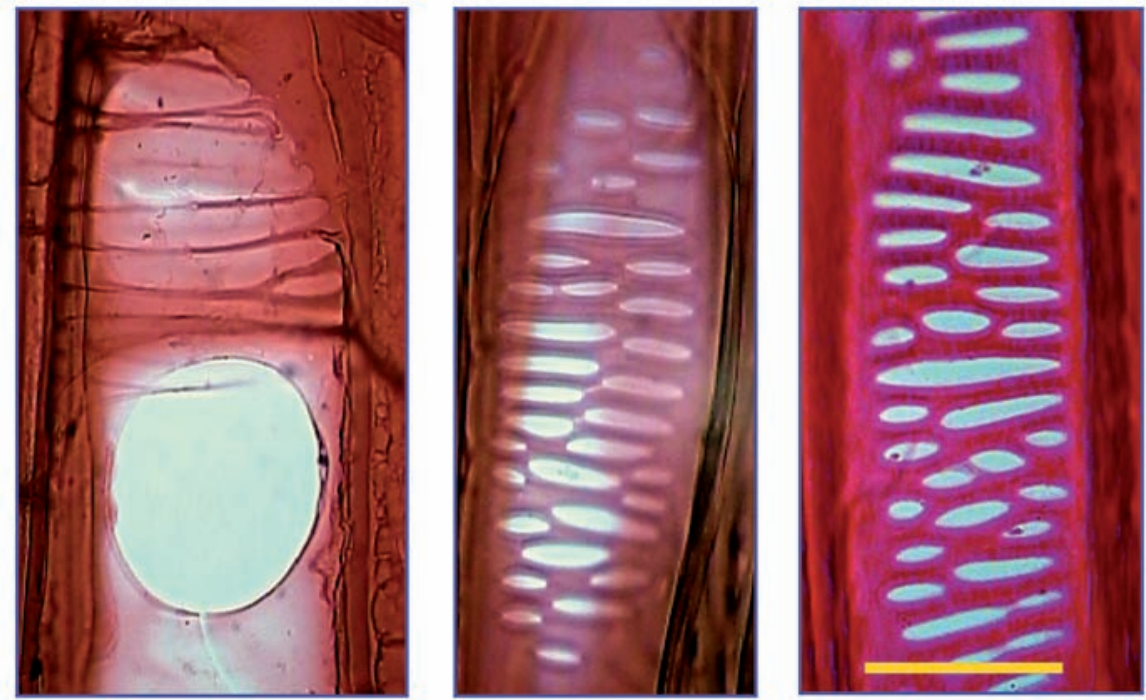

Figura 5: Otros tipos de perforaciones en cortes radiales. A: Combinación de placa en Laurelia sempervirens; B-C: Perforaciones con variadas formas en Laurelia sempervirens (B) y Laureliopsis philippiana (C). Escala: $30 \mu \mathrm{m}$ 


\section{CONCLUSIONES}

Las perforaciones escalariformes en las 10 especies de este estudio fueron clasificadas según el número de barras y espaciamiento entre éstas.

El análisis de las perforaciones escalariformes permitió observar variaciones y detalles de su estructura; encontrándose también otros tipos de perforaciones en algunas especies como Eucryphia cordifolia, Laurelia sempervirens y Laureliopsis philippiana.

Esta información espera ser un aporte para la identificación y conocimiento de la estructura anatómica de las maderas nativas de Chile.

\section{AGRADECIMIENTOS}

Este trabajo esta inserto en los programas de investigación "Mediciones biométricas de elementos anatómicos" y "Características macro y microscópicas de especies chilenas" desarrollados por el Laboratorio de Anatomía de la Madera, Departamento de Ingeniería de la Madera, Facultad de Ciencias Forestales, Universidad de Chile.

\section{BIBLIOGRAFÍA}

Baas, P.; Wheeler, E. A. 1996. Parallelism and reversibility in xylem evolution - A review. IAWA Journal 17 (4): 351-364.

Boureau, E. 1957. Anatomie végétale. Vol. 3. Presses Univ. France. Paris, 525-752 pp.

Carlquist, S. 1961. Comparative Plant Anatomy. Holt, Rinehart and Winston, INC. U.S.A. 146 pp.

Carlquist, S. 1988. Comparative Wood Anatomy. Systematic, Ecological and Evolutionary Aspects of Dicotyledon Wood. Springer Series in Wood Science. Editor Timell, T. E. 436 pp.

Diaz-vaz, J. E.; Poblete, H.; Juacida, R.; Devlieger, F. 2002. Maderas Comerciales de Chile. Chilean Comercial Woods. Marisa Cuneo Ediciones. Ed. 3. Valdivia Chile. 91 pp.

Diaz-vaz, J. E. 2003. Anatomía de Maderas. Marisa Cuneo Ediciones, Universidad Austral de Chile. Valdivia Chile. 151 pp.

Dickison, W. C. 1978. Comparative Anatomy of Eucryphiaceae. Amer. J. Bot. 65 (7): 722-735.

Ellerby, D. J.; Ennos, A. R. 1998. Resistances to fluid flow of model xylem vessels with simple and scalariform perforation plates. Journal of Experimental Botany 49: 979-985.

Hacke, U. G.; Sperry, J.S. 2001. Functional and ecological xylem anatomy. Perspect. Plant Ecol. Evol. Syst. 4(2): 97-115.

IAWA Committee. 1989. IAWA List of Microscopic Features for Hardwood Identification. IAWA Bull., n.s.10 (3): 219-332.

Jansen, S.; Baas, P.; Gasson, P.; Lens, F.; Smets, E. 2004. Variation in xylem structure from tropics to tundra: Evidence from vestured pits. Proceedings of the National Academy of Sciences 101(23):8833 - 8837 .

Metcalfe, C. R.; Chalk, L. 1983. Anatomy of the Dicotyledons. Vol.2. Clarendon Press, Oxford. 297 pp. 
Meylan, B. A.; Butterfield, B. G. 1975. Ocurrence of simple, multiple, and combination perforation plates in the vessels of New Zealand Woods. New Zeal. J. Bot. 13: 1-18.

Normand, D. 1972. Manuel d'Identification des Bois Commerciaux. Tome I.

Centre Technique Forestier Tropical. 171 pp.

Panshin, A. J.; Zeeuw, C. 1980. Textbook of wood technology. McGraw-Hill, New York, 772 pp.

Rancusi, M. H.; Nishida, M; Nishida, H. 1987. Xilotomy of Important Chilean Woods. In Nishida, M.(ed) Contributions to the botany in the Andes II. Ed. Academy Scientific Book, Tokio. 68 -158 pp.

Rudall, P. J. 1982. An inusual type of perforation plate in Canthium barbatum Seem.

(Rubiaceae) IAWA Bull. n.s. 3 (2): 127-129.

Schneider, E. L.; Carlquist, S. 2001. SEM Studies on vessel elements of Saururacae. IAWA Journal 22(2): $183-192$.

Segala, E.; Angyalossy-Alfonso, V. 2000. Ecological Trends in the Wood Anatomy of some of Brazilian Species.1.Growth Rings and Vessels. IAWA Journal 21(1): 3 - 30.

Schulte, P. J. 1999. Water flow trough a 20-pore perforation plate in vessels of Liquidambar Styraciflua. Journal of Experimental Botany 50: 1179 - 1187.

Wagemann, G. 1949. Maderas chilenas, contribución a su anatomía e identificación. Lilloa 16: 263 -375. 